\title{
Blood biomarkers are helpful in the prediction of response to chemoradiation in rectal cancer: a prospective, hypothesis driven study on patients with locally advanced rectal cancer
}

Citation for published version (APA):

Buijsen, J., van Stiphout, R. G., Menheere, P. P., Lammering, G., \& Lambin, P. (2014). Blood biomarkers are helpful in the prediction of response to chemoradiation in rectal cancer: a prospective, hypothesis driven study on patients with locally advanced rectal cancer. Radiotherapy and Oncology, 111(2), 237242. https://doi.org/10.1016/j.radonc.2014.03.006

Document status and date:

Published: 01/05/2014

DOI:

10.1016/j.radonc.2014.03.006

Document Version:

Publisher's PDF, also known as Version of record

\section{Document license:}

Taverne

Please check the document version of this publication:

- A submitted manuscript is the version of the article upon submission and before peer-review. There can be important differences between the submitted version and the official published version of record. People interested in the research are advised to contact the author for the final version of the publication, or visit the DOI to the publisher's website.

- The final author version and the galley proof are versions of the publication after peer review.

- The final published version features the final layout of the paper including the volume, issue and page numbers.

Link to publication

\footnotetext{
General rights rights.

- You may freely distribute the URL identifying the publication in the public portal. please follow below link for the End User Agreement:

www.umlib.nl/taverne-license

Take down policy

If you believe that this document breaches copyright please contact us at:

repository@maastrichtuniversity.nl

providing details and we will investigate your claim.
}

Copyright and moral rights for the publications made accessible in the public portal are retained by the authors and/or other copyright owners and it is a condition of accessing publications that users recognise and abide by the legal requirements associated with these

- Users may download and print one copy of any publication from the public portal for the purpose of private study or research.

- You may not further distribute the material or use it for any profit-making activity or commercial gain

If the publication is distributed under the terms of Article 25fa of the Dutch Copyright Act, indicated by the "Taverne" license above, 
Prediction in rectal cancer

\title{
Blood biomarkers are helpful in the prediction of response to chemoradiation in rectal cancer: A prospective, hypothesis driven study on patients with locally advanced rectal cancer
}

\author{
Jeroen Buijsen $^{\mathrm{a}, *, 1}$, Ruud G. van Stiphout ${ }^{\mathrm{a}, 1}$, Paul P.C.A. Menheere ${ }^{\mathrm{b}}$, Guido Lammering ${ }^{\mathrm{a}}$, Philippe Lambin ${ }^{\mathrm{a}}$

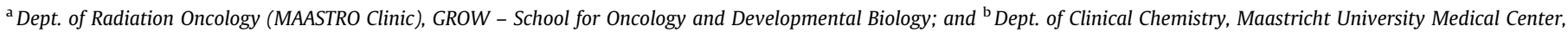 \\ The Netherlands
}

\section{A R T I C L E I N F O}

\section{Article history:}

Received 17 May 2013

Received in revised form 10 March 2014

Accepted 10 March 2014

Available online 16 April 2014

\section{Keywords:}

Rectal cancer

Chemoradiation

Blood biomarkers

Response prediction

\begin{abstract}
A B S T R A C T
Purpose/objective: Chemoradiation (CRT) has been shown to lead to downsizing of an important portion of rectal cancers. In order to tailor treatment at an earlier stage during treatment, predictive models are being developed. Adding blood biomarkers may be attractive for prediction, as they can be collected very easily and determined with excellent reproducibility in clinical practice. The hypothesis of this study was that blood biomarkers related to tumor load, hypoxia and inflammation can help to predict response to CRT in rectal cancer.

Material/methods: 295 patients with locally advanced rectal cancer who were planned to undergo CRT were prospectively entered into a biobank protocol (NCT01067872). Blood samples were drawn before start of CRT. Nine biomarkers were selected, based on a previously defined hypothesis, and measured in a standardized way by a certified lab: CEA, CA19-9, LDH, CRP, IL-6, IL-8, CA IX, osteopontin and 25$\mathrm{OH}$-vitamin D. Outcome was analyzed in two ways: pCR vs. non-pCR and responders (defined as ypT02N0) vs. non-responders (all other ypTN stages).

Results: 276 patients could be analyzed. $20.7 \%$ developed a pCR and $47.1 \%$ were classified as responders. In univariate analysis CEA $(p=0.001)$ and osteopontin $(p=0.012)$ were significant predictors for pCR. Taking response as outcome CEA $(p<0.001)$, IL- $8(p<0.001)$ and osteopontin $(p=0.004)$ were significant predictors. In multivariate analysis CEA was the strongest predictor for pCR (OR 0.92, $p=0.019)$ and CEA and IL- 8 predicted for response (OR 0.97, $p=0.029$ and OR 0.94, $p=0.036$ ). The model based on biomarkers only had an AUC of 0.65 for pCR and 0.68 for response; the strongest model included clinical data, PET-data and biomarkers and had an AUC of 0.81 for PCR and 0.78 for response.

Conclusion: CEA and IL-8 were identified as predictive biomarkers for tumor response and PCR after CRT in rectal cancer. Incorporation of these blood biomarkers leads to an additional accuracy of earlier developed prediction models using clinical variables and PET-information. The new model could help to an early adaptation of treatment in rectal cancer patients.
\end{abstract}

(c) 2014 Elsevier Ireland Ltd. All rights reserved. Radiotherapy and Oncology 111 (2014) 237-242
Combined treatment is the cornerstone of rectal cancer treatment. In case of locally advanced rectal cancer, defined as a tumor with a predicted positive circumferential resection margin (CRM) or four or more positive lymph nodes, chemoradiotherapy (CRT) has become the standard of care [1]. Pathological complete response (pCR) rates typically lie between 15\% and 20\% after CRT $[2,3]$ depending on the radiotherapy dose given and the interval between CRT and surgery. The group of patients that develop a $\mathrm{pCR}$ is of particular interest, because they have a better prognosis

\footnotetext{
* Corresponding author. Address: MAASTRO Clinic, PO Box 1345, 6201 BH Maastricht, The Netherlands.

E-mail address: jeroen.buijsen@maastro.nl (J. Buijsen).

1 These authors contributed equally to this article.
}

[2] and may be offered less invasive surgery [4] or surgery may be completely omitted $[5,6]$. Therefore it would be an advantage if the pCR rate could be increased. There are several possible strategies, of which early response prediction during CRT, leading to treatment adaptation, is very attractive.

In the past, clinical parameters as well as information from PETscans before and during treatment have been found to be predictive for treatment outcome [7-9]. A prediction model based on tumor length, cT- and cN-stage had a predictive performance of 0.61 as expressed by the area under the curve (AUC) of the receiver operating characteristic (ROC) curve. A second model including maximal standardized uptake value (SUV) of the tumor derived from a PET-scan before the start of treatment, maximal tumor diameter as measured on PET-scan, tumor location and cN-stage, 
resulted in an AUC of 0.68 [9]. PET-scan after 2 weeks of CRT has been shown to be very predictive for response (tumor regression grade (TRG) 1-2 vs. TRG 3-5 according to Mandard) [8].

It is attractive to consider the addition of blood biomarkers to these predictors, since samples can be collected easily, are relatively cheap to measure, and they contain information about different aspects of tumor biology. Furthermore, they can be measured accurately and precisely using standardized methods. Reports on the predictive value of blood biomarkers are limited to studies mainly evaluating 1 or 2 biomarkers [7,10-13]. The most studied biomarker for response to CRT in rectal cancer is carcinoembryonic antigen (CEA) and one study analyzed the predictive value of osteopontin and interleukin-6. Based on these data combined with data of prognostic studies in colorectal cancer and our experience with a blood biomarker model in lung cancer [14], we decided to include 9 biomarkers. CEA and CA19-9 are related to tumor load, interleukin-6 and -8 (IL-6 and IL-8) and C-reactive protein (CRP) are markers of inflammation, lactate dehydrogenase (LDH) is a marker of cell death, carbonic anhydrase IX (CA IX) and osteopontin are hypoxia markers and 25-OH-vitamin D may induce growth arrest and apoptosis of tumor cells.

In this prospective study we tested the hypothesis that these biomarkers are helpful in the prediction of response to CRT in rectal cancer.

\section{Methods and materials}

\section{Patient population}

All patients who were treated according to the protocol "chemoradiation, rectal cancer, 50.4 Gy" between January 2005 and December 2009 and who gave written informed consent for inclusion in the biobank protocol (NCT01067872) and from whom blood samples were collected were included in this study. This query resulted in 295 patients. Nineteen patients were ineligible for different reasons ( 1 patient was treated with short course radiotherapy $5 \times 5 \mathrm{~Gy}, 5$ patients were not operated on and entered in a wait and see study so no ypTN stage could be determined, 2 patients underwent a TEM resection so no ypN stage was available, 6 patients had metastases and were treated with palliative intent, 1 patient died during treatment, in 1 patient all biobank material was hemolytic and in 3 patients the pathology report could not be retrieved), resulting in 276 patients for analysis. In 9 patients there were technical problems with the biomarker measurements, so that 267 patients were available for the biomarker analysis. Locally advanced disease was defined as a distal T3 tumor and/or N2 status and/or a mid- or upper-rectal tumor with a predicted circumferential resection margin $<2 \mathrm{~mm}$, or any T4 tumor. Locoregional staging for clinical tumor and nodal stage was based on MRI. In 198 patients for whom biomarkers were available a PET-CT was made for radiotherapy planning. Treatment consisted of 28 fractions of $1.8 \mathrm{~Gy}$ on the primary tumor, mesorectum, presacral area and external iliac lymph nodes in combination with capecitabine $825 \mathrm{mg} / \mathrm{m}^{2}$ BID. Fourteen patients did not receive a dose of $50.4 \mathrm{~Gy}$. In 12 patients treatment had to be discontinued because of toxicity and in 2 patients a lower total dose was prescribed because of radiotherapy in the past. Patients were operated on 8-10 weeks after the end of CRT. Pathology reports were collected from the referring hospitals.

\section{Blood samples}

Blood samples were collected before the start of treatment. Samples were processed and stored using a standard protocol. All biomarkers were measured in serum, except for osteopontin and CA IX, which were measured in EDTA plasma. Biomarker measure- ments were done in one single, certified laboratory, using commercially available kits. All samples were analyzed simultaneously. Measurements were performed using the following kits: CEA was measured using a solid-phase, two-site sequential chemoluminescent immunometric assay (Siemens Medical Solutions Diagnostics, LA, USA), IL-6 and IL-8 were determined with a solid phase, enzyme labeled, chemoluminescence sequential immunometric assay (Siemens Medical Solutions Diagnostics, LA, USA). LDH (Beckman Coulter, Fullerton, CA), CRP (Beckman, Coulter Fullerton, CA) and CA 19-9 have been determined on Brahms Kryptor (Brahms, ThermoFisher, Hennigsdorf, Germany) with a sandwich immuno-fluorescent assay. 25-OH-vitamin-D was measured with a commercially available radioimmunoassay (IDS, Frankfurt am Main, Germany). CA IX was measured by an enzyme-linked immunosorbent assay (Wilex (OncogenScience), Cambridge, MA, USA), and OPN was measured by an enzyme-linked immunosorbent assay (Quantikine Human Osteopontin Immuno assay; R\&D Systems, Minneapolis, MN, USA). OPN, 25-OH-vitamin-D and CA IX were measured using manual methods in duplicate. All other biomarkers were measured in singletons.

\section{Statistical analysis}

Primary endpoint in this study was PCR defined as the absence of any tumor cells in the operative pathologic specimen, at the primary site, or in lymph node regions. It was hypothesized that the addition of biomarkers would lead to an increase of the AUC from 0.7 to 0.8 . Assuming a correlation between the models of 0.6 a sample of 57 patients with PCR and 219 without pCR achieves a power of 0.74 to detect a difference of 0.1 between the model with and without biomarkers. An alternative endpoint was good responders, defined as ypT0-2N0, versus poor responders, defined as ypT3 and/or ypN1-2. Missing values were completed using expectation-maximation imputation. Correlations between biomarkers were analyzed by calculating Spearman's rho. Because the biomarkers showed a skewed distribution, the Mann-Whitney $U$ test was used to determine significant univariate predictors of response. Logistic regression analysis was used to identify the significant multivariate predictors of response. The next step was to combine blood biomarkers with clinical data and PET parameters. For this analysis clinical and PET-variables were selected manually, based on an earlier predictive model for PCR [9]. The included clinical variables were tumor length, clinical $\mathrm{T}$ and $\mathrm{N}$ stage, all based on MRI. Included PET-features were maximum SUV, pretreatment metabolic volume and maximum diameter. The two latter variables were measured using a source-to-background ratio method as has been described earlier [15]. ROC curves were constructed and the AUC was calculated. In order to approximate the true AUC and calculate confidence intervals bootstrapping $(n=1000)$ was used. A perfect prediction model results in an AUC of 1.0, while an AUC of 0.5 indicates that response is predicted correctly in $50 \%$ of cases (i.e. as good as chance). Statistical analyses were performed using Matlab, release 2010b (The MathWorks, Natick, MA).

\section{Ethics}

The biobank study was conducted according to the Dutch law and approved by the local medical ethics committee. All patients gave written informed consent before collection of the blood samples.

\section{Results}

The patient characteristics are shown in Table 1 . The majority of patients had a tumor penetrating through the bowel wall and predicted positive lymph nodes on MR. In the total database $20.7 \%$ of 
Table 1

Patient characteristics $(N=276)$.

\begin{tabular}{|c|c|c|}
\hline Characteristic & $N$ & [\%] \\
\hline \multicolumn{3}{|l|}{ Age [years] } \\
\hline Median & 65.8 & \\
\hline Range & $23.0-92.2$ & \\
\hline \multicolumn{3}{|l|}{ Gender } \\
\hline Male & 179 & [64.9] \\
\hline Female & 97 & [35.1] \\
\hline \multicolumn{3}{|c|}{ Clinical tumor stage (cT) } \\
\hline 2 & 26 & {$[9.4]$} \\
\hline 3 & 207 & [75.0] \\
\hline 4 & 43 & [15.6] \\
\hline \multicolumn{3}{|c|}{ Clinical nodal stage $(\mathrm{cN})$} \\
\hline 0 & 47 & [17.0] \\
\hline 1 & 114 & [41.3] \\
\hline 2 & 115 & [41.7] \\
\hline \multicolumn{3}{|c|}{ WHO performance index } \\
\hline 0 & 218 & [79.0] \\
\hline 1 & 53 & [19.2] \\
\hline 2 & 5 & {$[1.8]$} \\
\hline \multicolumn{3}{|c|}{ Tumor length $[\mathrm{cm}]$} \\
\hline Median & 5.0 & \\
\hline Range & $2.0-13.0$ & \\
\hline \multicolumn{3}{|l|}{ pCR (ypTONO) } \\
\hline No & 219 & [79.3] \\
\hline Yes & 57 & [20.7] \\
\hline \multicolumn{3}{|c|}{ Good response (ypT012N0) } \\
\hline No & 146 & [52.9] \\
\hline Yes & 130 & [47.1] \\
\hline
\end{tabular}

patients developed a PCR and $47.1 \%$ of patients were classified as responder. Table 2 shows the results of the biomarker measurements for the different outcome groups, as well as the PET-parameters that were included in the model. In general lower serum levels for blood biomarkers were seen in the poor responding groups (except for 25-OH-vitamin-D). Additional analysis (not shown in the table) revealed significant positive correlations between IL-6, IL-8, CRP and osteopontin and between CEA and CA19-9.

\section{Univariate analysis}

Univariate analysis indicated that CEA and osteopontin were significant predictors for $\mathrm{pCR}$ ( $p=0.001, p=0.012$ respectively) and that CEA, IL-8 and osteopontin were significant predictors for response $(p<0.001, p<0.001, p=0.004$, respectively) as shown in Table 3 (Supplementary data). Lower serum levels of these markers correlated with a higher chance of response to chemoradiation. Of the clinical parameters clinical $\mathrm{N}$-stage was predictive for $\mathrm{pCR}$ $(p=0.026)$ and response $(p=0.001)$ in univariate analysis and tumor length $(p=0.02)$ and clinical T-stage $(p=0.004)$ for response only. The pre-treatment metabolic volume and maximum diameter based on PET were predictive for both outcome measures ( $p=0.016$ and 0.009 for $\mathrm{pCR}$ and $p=0.006$ and 0.005 for response respectively).

\section{Multivariate biomarker model}

Table 3 (Supplementary data) shows the results of the multivariate analysis for the total set of parameters as well as a selection of biomarkers and clinical and PET-parameters. In the complete set of parameters IL-8 was the only significant predictor for response $(p=0.05)$, while osteopontin was borderline significant for $\mathrm{pCR}$ prediction $(p=0.056)$. As a next step a manual selection of the most promising predictors was made. Blood biomarkers that had a significant predictive value in either univariate or multivariate analysis were included and IL-6, although not significant, was included because it had a predictive value in a prognostic model for lung cancer [14]. In this selection of biomarkers consisting of CEA, IL-6, IL-8 and osteopontin, CEA was the only significant predictor of $\mathrm{pCR}$ in multivariate analysis $(p=0.019)$ and CEA and IL-8 significantly predicted response $(p=0.029, p=0.021$ respectively). Including all biomarkers resulted in an AUC of 0.65 (95\% CI $0.57-0.73$ ) for pCR prediction and 0.68 (95\% 0.61-0.75) for response prediction. Table 3 (Supplementary data) shows the odds ratios for all tested biomarkers as well as the clinical and PET-based parameters.

\section{Combination of blood biomarkers with clinical and PET data}

The biomarker selection was then added to the parameters that were predictive for response in an externally validated prediction model based on clinical and PET-scan data [9]. The final model consisted of eight variables: tumor length, clinical T stage, clinical $\mathrm{N}$ stage, CEA, IL-6, IL-8, osteopontin, and maximal SUV on PET before start of treatment. In the current dataset tumor length was not a significant predictor for response to chemoradiation, but $\mathrm{cT}$ and $\mathrm{cN}$ were. Maximal SUV was only predictive for response, not for PCR.

In Fig. 1 the ROC curves for the combined models based on biomarkers and clinical data (Fig. $1 \mathrm{~A}$ and a) and biomarkers, clinical data and PET information (Fig. 1B and b) are depicted, as well as the resulting AUCs (Fig. 1C and c). The model based on biomarkers only resulted in an AUC that was more or less comparable to the clinical model. The AUC of the clinical model was 0.64 (95\% CI 0.56-0.71) for pCR and 0.66 (95\% CI 0.60-0.72) for response. Combining clinical parameters and biomarkers (AUC 0.69 (95\% CI 0.62-0.77) for pCR and 0.73 (95\% CI 0.65-0.79) for response) made the model stronger than the model based on biomarkers only or clinical data only. This

Table 2

Biomarker levels and PET parameters (average \pm standard deviation) compared for the subpopulations of pCR vs. no pCR and good response vs. no good response.

\begin{tabular}{|c|c|c|c|c|c|}
\hline & & \multicolumn{2}{|l|}{ pCR (ypTON0) } & \multicolumn{2}{|c|}{ Good response (ypT012N0) } \\
\hline & & No & Yes & No & Yes \\
\hline \multirow{9}{*}{$\begin{array}{l}\text { Biomarkers } \\
(N=267)\end{array}$} & CEA & $14.7 \pm 28.4$ & $8.7 \pm 22.9$ & $18.1 \pm 33$ & $8.5 \pm 18.5$ \\
\hline & IL-6 & $4.0 \pm 8.4$ & $2.9 \pm 2.7$ & $4.8 \pm 10.1$ & $2.7 \pm 2.4$ \\
\hline & IL-8 & $15.8 \pm 9$ & $14.0 \pm 7.4$ & $17.3 \pm 10$ & $13.4 \pm 6.4$ \\
\hline & LDH & $181.3 \pm 43.8$ & $176.2 \pm 33.9$ & $181.9 \pm 46.7$ & $178.5 \pm 36.1$ \\
\hline & CRP & $11.5 \pm 24.5$ & $7.8 \pm 8$ & $13.8 \pm 29.1$ & $7.5 \pm 9.2$ \\
\hline & CA 19-9 & $26.9 \pm 29.1$ & $25.1 \pm 31.9$ & $28.3 \pm 28.8$ & $24.6 \pm 30.6$ \\
\hline & vitD-25 & $53.2 \pm 20.9$ & $55.5 \pm 18.8$ & $52.6 \pm 22.4$ & $54.8 \pm 18.2$ \\
\hline & CA-9 & $282.5 \pm 275$ & $274.4 \pm 302.9$ & $282.0 \pm 238.4$ & $279.6 \pm 320.9$ \\
\hline & OPN & $79.2 \pm 28.9$ & $68.2 \pm 16$ & $81.6 \pm 30.9$ & $72 \pm 21.3$ \\
\hline \multirow{4}{*}{$\begin{array}{l}\text { PET parameters } \\
(N=198)\end{array}$} & $\mathrm{SUV}_{\max }$ & $15.3 \pm 6.3$ & $13.6 \pm 5.2$ & $14.3 \pm 5.6$ & $15.6 \pm 6.7$ \\
\hline & $\mathrm{SUV}_{\text {mean }}$ & $8.2 \pm 3.1$ & $7.4 \pm 2.7$ & $7.8 \pm 2.7$ & $8.4 \pm 3.3$ \\
\hline & MTV [cc] & $33.8 \pm 33.5$ & $25 \pm 20.2$ & $37.6 \pm 39.1$ & $25.7 \pm 17.6$ \\
\hline & Max diameter $[\mathrm{cm}]$ & $6.5 \pm 1.9$ & $5.8 \pm 1.6$ & $6.7 \pm 2.1$ & $5.9 \pm 1.4$ \\
\hline
\end{tabular}



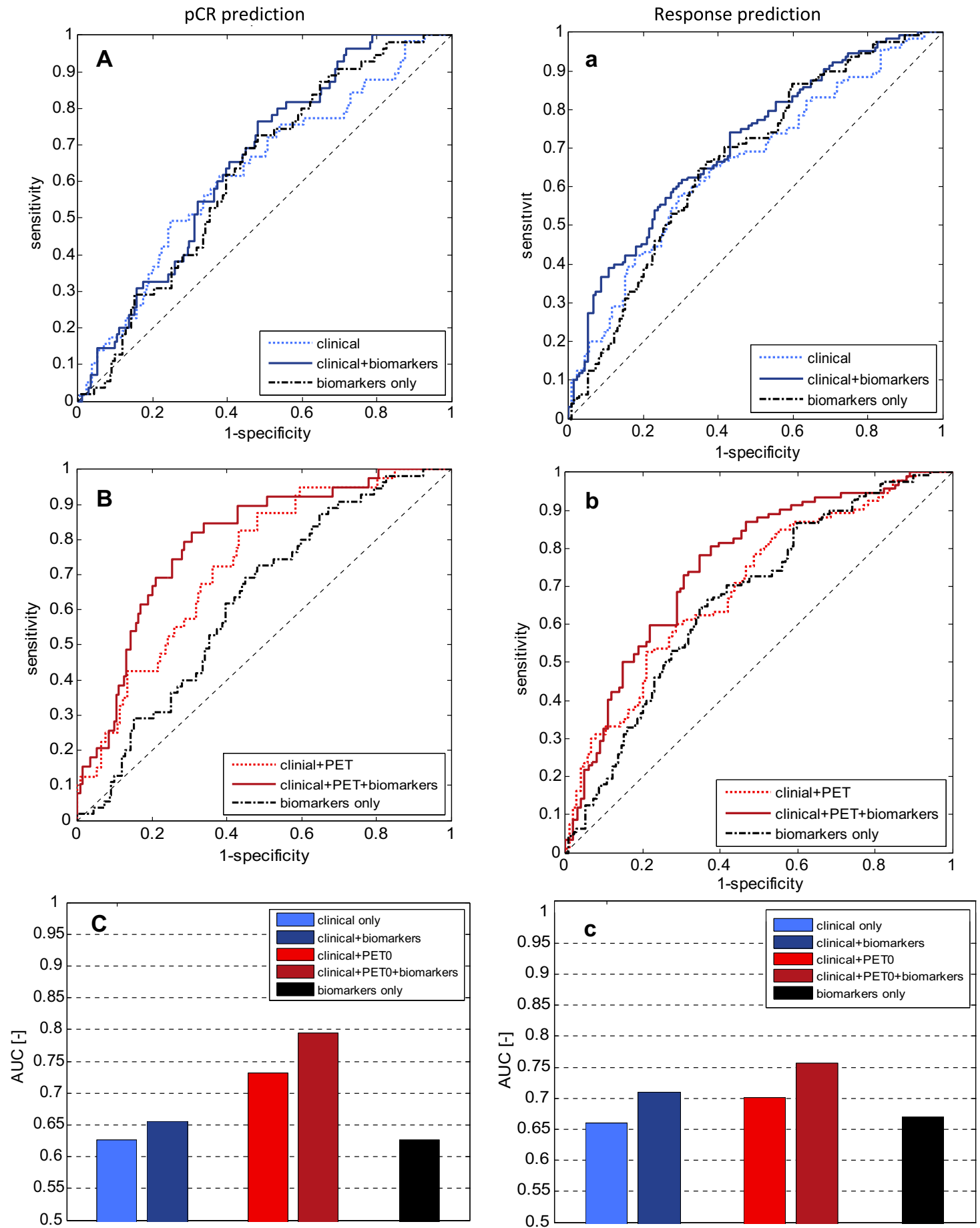

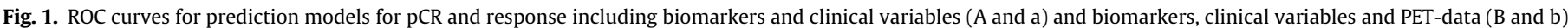
and resulting AUC for the different prediction models ( $\mathrm{C}$ and $\mathrm{c}$ ).

effect was most pronounced for prediction of response. Addition of information from a PET-scan acquired before the start of treatment leads to the strongest models for the prediction of both PCR and response, resulting in AUCs of 0.81 (95\% CI 0.73-0.88) for pCR and 0.78 (95\% CI 0.71-0.85) for response.

The data of the combined prediction model was used to build a nomogram, which is depicted in Fig. 2. This nomogram can be used online at www.predictcancer.org. Because this nomogram has not yet been externally validated, it should be regarded as hypothesis generating.

\section{Discussion}

To the best of our knowledge this is the first prospective study evaluating the predictive value of a broad range of blood biomarkers analyzed in a standardized way for response prediction to CRT in rectal cancer. CEA turned out to be a significant predictor for $\mathrm{pCR}$ and CEA and IL- 8 were predictive for response. Including these blood biomarkers in models based on clinical parameters and PET-based parameters resulted in an increased performance of the prediction models. 


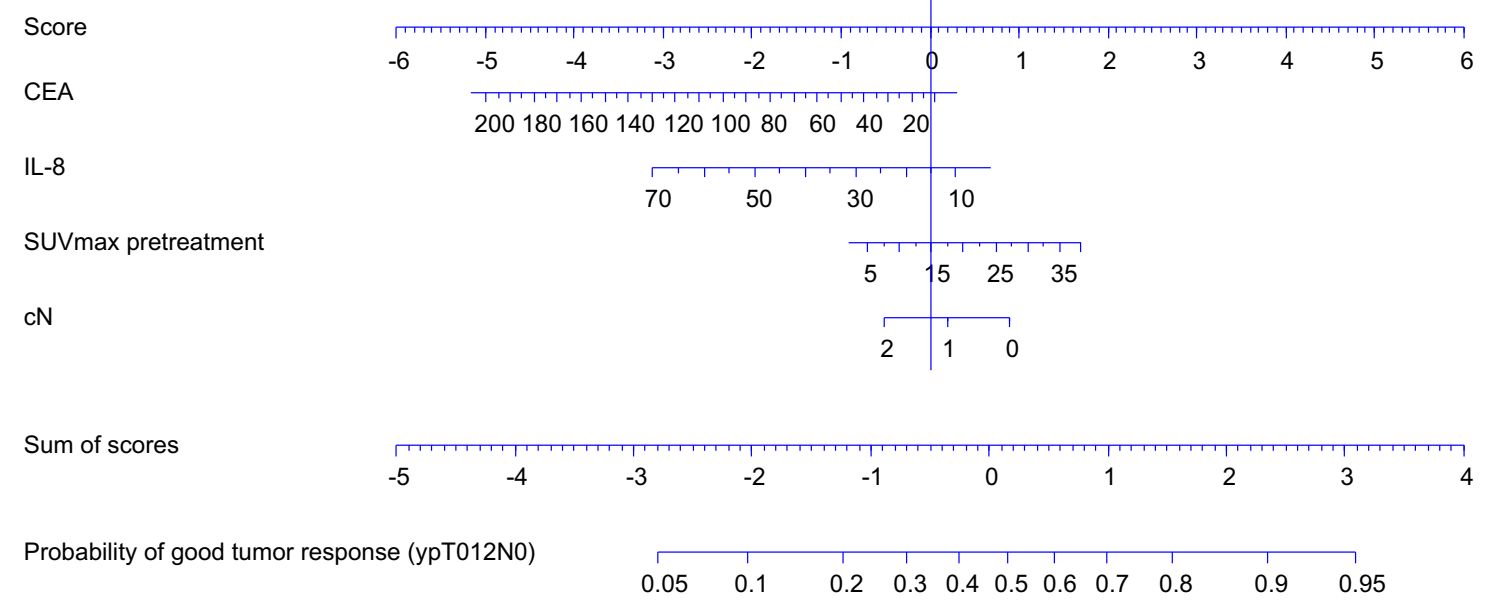

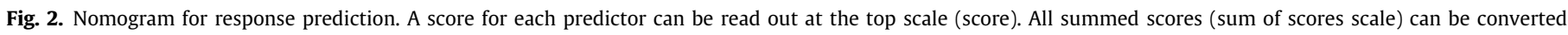
directly to the probability of response.

Osteopontin is a glycophosphoprotein secreted by different cell types, that has been shown to be associated not only with malignancies but also with acute and chronic inflammatory processes. It influences adhesion, migration, invasion, chemotaxis and cell survival [16] and is a marker of tumor aggressiveness and early progression [17]. Although the role of osteopontin as a prognostic factor has been studied extensively in a broad range of solid tumors, only one study looked at the predictive value of osteopontin levels and response to CRT in rectal cancer. Debucquoy et al. found an association between lower osteopontin levels and better response in 30 rectal cancer patients, which is in line with our findings, but this did not reach statistical significance [13]. In our patient group it was a significant predictor in univariate analysis, but it lost significance in multivariate analysis. This could be explained by the fact that osteopontin and IL- 8 showed a positive correlation.

CEA is an antigen produced by the normal fetus and only in very low concentrations by normal cells of the adult body. In the tumor environment it plays a role in intercellular recognition and attachment. It has been shown to be of prognostic value in colorectal cancer independent of clinical stage and differentiation grade [18]. Although preoperative CEA levels cannot be used to make treatment decisions in colorectal cancer, they consistently have a prognostic value. Patients having a CEA level $>5 \mu \mathrm{g} / \mathrm{l}$ have a significant worse disease free and overall survival than patients with lower CEA levels. Three studies looked at the predictive value of CEA levels for response to CRT in rectal cancer. Das et al. studied a group of 562 rectal cancer patients treated with CRT [7]. In their patient group CEA was only predictive for PCR in univariate analysis. However, they chose to dichotomize CEA levels (below and above $2.5 \mu \mathrm{g} / \mathrm{l})$, while in our model CEA was incorporated as a continuous variable. Yoon et al. did an analysis in a group of 351 rectal cancer patients [12]. In multivariate analysis CEA levels $\leqslant 5 \mu \mathrm{g} / \mathrm{l}$ were predictive for downstaging and complete regression. Park et al. did a retrospective analysis in 352 rectal cancer patients [11]. CEA was analyzed as a continuous variable and had a significant predictive value for responders vs. non-responders. A higher CEA was an independent predictor of poor response to CRT and a worse disease free survival.

IL-8 is a pro-inflammatory chemokine that plays a role in attracting neutrophils. Through the activation of phosphatidyl-inositol-3-kinase (PI3-K) and phospholipase C it can activate the Akt/ mTOR and Raf/Mek/Erk pathways, leading to the promotion of angiogenesis, proliferation and survival and the migration of cancer cells [19]. Polymorphisms in IL-8 have been described to be related to an increased risk of recurrence in rectal cancer [20] and the risk of nodal involvement [21], indicating a possible relationship with tumor biology of IL-8 in rectal cancer. However, until now there are no reports of a potential value of IL- 8 in the prediction of response to CRT.

In this study response was measured in two ways: $\mathrm{PCR}$ and ypT0-2N0. Of these 2 endpoints PCR is the most robust, although the definition of pCR can be difficult. In this cohort routine pathological examination was performed and reported in a standardized way. A recent comparison between routine pathological examination and additional step sections in resection specimens showing no viable tumor cells at initial examination, showed no differences in outcome [22]. Furthermore, pooled analysis of a large series of patients included in different studies, showed a clear prognostic value of pCR after CRT for long-term outcome, even if pooled from different studies, indicating that pCR as scored in routine pathology procedures is a valuable endpoint [2]. The most frequently used method to distinguish responders from non-responders is by means of a tumor regression grade (TRG). However, for this cohort TRG was not scored routinely and we chose to use ypT0-2N0 as definition of response. It makes sense to predict the group of good responders, because they could be suitable for less invasive surgery, like transanal resection or TEM-surgery.

Blood biomarkers give information about tumor biology in an indirect way. A more direct insight can be gained from genetic alterations within the tumor. An overview of these molecular biomarkers is given in the review of Kuremsky et al. [23]. A possible problem related to molecular biomarkers is the heterogeneity in tumors, making it necessary to collect a representative sampling of tumor material and the invasive procedure needed to collect material. Blood biomarkers have the advantage that they are easy to collect and that they provide information about the "average" tumor. This makes blood biomarkers useful for daily practice. However translation of the results of this study should be done cautiously, because all biomarkers in this study were measured in a standardized way in one single laboratory, using the same kits. Less thorough and sensitive procedures might influence negatively the added value of biomarkers.

Ideally response prediction takes place before the start of a treatment, so that a patient can be offered the treatment with the highest success rate. In the case of response prediction for rectal cancer, clinical factors and pre-treatment PET-scan have been shown to have predictive value before the start of treatment [9], 
but the performance of predictive models based exclusively on pre-treatment data typically lies between 0.65 and 0.70 . The predictive value of biomarkers only is in the same range, but the combination of biomarkers and other pre-treatment data results in a stronger prediction model.

The data presented here can be seen as a proof of principle that biomarkers contain predictive information for rectal cancer, but external validation of this prediction model is necessary for a better estimation of the performance and reproducibility of the model [24]. For use in clinical practice a stronger performance is desirable. A possibility to strengthen the predictive model is to incorporate response data obtained early during CRT. If this time point lies early in the treatment course, it is still possible to modify treatment. For PET-CT it has already been shown that changes in glucose metabolism after 2 weeks of CRT resulted in a stronger prediction model [8]. An intriguing question is whether early changes in blood biomarker levels during CRT can further enhance the performance of this model. This question will be the subject of future research.

In conclusion, pre-treatment CEA levels help to predict pCR after CRT for rectal cancer and CEA and IL- 8 levels are helpful in the prediction of response to CRT. These blood biomarkers have added value to earlier published prediction models based on pretreatment clinical- and PET-data and can be used in decision support systems for tailored therapy [24].

\section{Acknowledgements}

We acknowledge financial support from the CTMM framework (AIRFORCE project, $\mathrm{n}^{\circ}$ 030-103), EU 7th framework program (METOXIA, EURECA), euroCAT (IVA Interreg, www.eurocat.info), Radiomics (NIH, USA), EU IMI program (QuIC-ConCePT), NIH-QIN (Radiomics of NSCLC U01 CA143062) and the Dutch Cancer Society (KWF UM 2011-5020, KWF UM 2009-4454). The authors wish to thank Nancy Hendrix and Jos Wiersma from the department of clinical chemistry who performed all biomarker analyses.

\section{Appendix A. Supplementary data}

Supplementary data associated with this article can be found, in the online version, at http://dx.doi.org/10.1016/j.radonc.2014.03. 006.

\section{References}

[1] Valentini V, Aristei C, Glimelius B, et al. Multidisciplinary rectal cancer management: 2nd European rectal cancer consensus conference (EURECACC2). Radiother Oncol 2009;92:148-63.

[2] Maas M, Nelemans PJ, Valentini V, et al. Long-term outcome in patients with a pathological complete response after chemoradiation for rectal cancer: a pooled analysis of individual patient data. Lancet Oncol 2010;11:835-44.
[3] Zorcolo L, Rosman AS, Restivo A, et al. Complete pathologic response after combined modality treatment for rectal cancer and long-term survival: a meta-analysis. Ann Surg Oncol 2012.

[4] Bokkerink GM, de Graaf EJ, Punt CJ, et al. The CARTS study: chemoradiation therapy for rectal cancer in the distal rectum followed by organ-sparing transanal endoscopic microsurgery. BMC Surg 2011;11:34.

[5] Maas M, Beets-Tan RG, Lambregts DM, et al. Wait-and-see policy for clinical complete responders after chemoradiation for rectal cancer. J Clin Oncol 2011;29:4633-40.

[6] Glynne-Jones R, Hughes R. Critical appraisal of the 'wait and see' approach in rectal cancer for clinical complete responders after chemoradiation. Br J Surg 2012;99:897-909.

[7] Das P, Skibber JM, Rodriguez-Bigas MA, et al. Predictors of tumor response and downstaging in patients who receive preoperative chemoradiation for rectal cancer. Cancer 2007;109:1750-5.

[8] Janssen MH, Ollers MC, van Stiphout RG, et al. PET-based treatment response evaluation in rectal cancer: prediction and validation. Int J Radiat Oncol Biol Phys 2012;82:871-6.

[9] van Stiphout RG, Lammering G, Buijsen J, et al. Development and external validation of a predictive model for pathological complete response of rectal cancer patients including sequential PET-CT imaging. Radiother Oncol 2011;98:126-33.

[10] Lu K, Zhu Y, Sheng L, Liu L, Shen L, Wei Q. Serum fibrinogen level predicts the therapeutic response and prognosis in patients with locally advanced rectal cancer. Hepatogastroenterology 2011;58:1507-10.

[11] Park JW, Lim SB, Kim DY, et al. Carcinoembryonic antigen as a predictor of pathologic response and a prognostic factor in locally advanced rectal cancer patients treated with preoperative chemoradiotherapy and surgery. Int J Radiat Oncol Biol Phys 2009;74:810-7.

[12] Yoon SM, Kim DY, Kim TH, et al. Clinical parameters predicting pathologic tumor response after preoperative chemoradiotherapy for rectal cancer. Int J Radiat Oncol Biol Phys 2007;69:1167-72.

[13] Debucquoy A, Goethals L, Geboes K, Roels S, Mc Bride WH, Haustermans K. Molecular responses of rectal cancer to preoperative chemoradiation. Radiother Oncol 2006;80:172-7.

[14] Dehing-Oberije C, Aerts $\mathrm{H}$, Yu S, et al. Development and validation of a prognostic model using blood biomarker information for prediction of survival of non-small-cell lung cancer patients treated with combined chemotherapy and radiation or radiotherapy alone (NCT00181519, NCT00573040, and NCT00572325). Int J Radiat Oncol Biol Phys 2011:81:360-8.

[15] Buijsen J, van den Bogaard J, Janssen MH, et al. FDG-PET provides the best correlation with the tumor specimen compared to MRI and CT in rectal cancer. Radiother Oncol 2011;98:270-6.

[16] Anborgh PH, Mutrie JC, Tuck AB, Chambers AF. Role of the metastasispromoting protein osteopontin in the tumour microenvironment. J Cell Mol Med 2010;14:2037-44.

[17] Weber GF, Lett GS, Haubein NC. Osteopontin is a marker for cancer aggressiveness and patient survival. Br J Cancer 2010;103:861-9.

[18] Goldstein MJ, Mitchell EP. Carcinoembryonic antigen in the staging and follow-up of patients with colorectal cancer. Cancer Invest 2005;23:338-51.

[19] Waugh DJ, Wilson C. The interleukin-8 pathway in cancer. Clin Cancer Res 2008;14:6735-41.

[20] Gordon MA, Gil J, Lu B, et al. Genomic profiling associated with recurrence in patients with rectal cancer treated with chemoradiation. Pharmacogenomics 2006;7:67-88.

[21] Kalady MF, Coffey JC, Dejulius K, Jarrar A, Church JM. High-throughput arrays identify distinct genetic profiles associated with lymph node involvement in rectal cancer. Dis Colon Rectum 2012;55:628-39.

[22] Park SY, Chang HJ, Kim DY, et al. Is step section necessary for determination of complete pathological response in rectal cancer patients treated with preoperative chemoradiotherapy? Histopathology 2011;59:650-9.

[23] Kuremsky JG, Tepper JE, McLeod HL. Biomarkers for response to neoadjuvant chemoradiation for rectal cancer. Int J Radiat Oncol Biol Phys 2009;74:673-88.

[24] Lambin P, van Stiphout RG, Starmans MH, et al. Predicting outcomes in radiation oncology-multifactorial decision support systems. Nat Rev Clin Oncol 2012. 